\title{
Between- and Within-Individual Variation of Maternal Thyroid Hormone Deposition in Wild Great Tits (Parus major)
}

\author{
Bin-Yan Hsu, ${ }^{1, \star}$ Irene Verhagen, ${ }^{2}$ Phillip Gienapp, ${ }^{2}$ Veerle M. Darras, ${ }^{3}$ Marcel E. Visser, ${ }^{2}$ \\ and Suvi Ruuskanen ${ }^{1,2}$
}

1. Section of Ecology, Department of Biology, University of Turku, Turku, Finland; 2. Department of Animal Ecology, Netherlands Institute of Ecology, Wageningen, The Netherlands; 3. Laboratory of Comparative Endocrinology, Department of Biology, Katholieke Universiteit Leuven, Leuven, Belgium

Submitted December 21, 2018; Accepted March 14, 2019; Electronically published August 9, 2019

Online enhancements: appendix. Dryad data: https://doi.org/10.5061/dryad.kp524f5.

\begin{abstract}
Maternal hormones are often considered a mediator of anticipatory maternal effects; namely, mothers adjust maternal hormone transfer to prepare the offspring for the anticipated environment. The flexibility for mothers to adjust hormone transfer is therefore a prerequisite for such anticipatory maternal effects. Nevertheless, previous studies have focused only on the average differences of maternal hormone transfer between groups and neglected the substantial individual variation, despite the fact that individual plasticity in maternal hormone transfer is actually the central assumption. In this study, we studied the between- and within-individual variation of maternal thyroid hormones (THs) in egg yolk of wild great tits (Parus major) and estimated the individual plasticity of maternal yolk THs across environmental temperature, clutch initiation dates, and egg laying order using linear mixed effects models. Interestingly, our models provide statistical evidence that the two main $\mathrm{THs}$ - the main biologically active hormone $\mathrm{T} 3$ and $\mathrm{T} 4$, which is mostly considered a prohormone - exhibited different variation patterns. Yolk T3 showed significant between-individual variation on the average levels, in line with its previously reported moderate heritability. Yolk T4, however, showed significant between-clutch variation in the pattern over the laying sequence, suggesting a great within-individual plasticity. Our findings suggest that the role and function of the hormone within the endocrine axis likely influences its flexibility to respond to environmental change. Whether the flexibility of T4 deposition brings a fitness advantage should be examined along with its potential effects on offspring, which remain to be further investigated.
\end{abstract}

Keywords: great tits, individual variation, maternal hormones, multilevel linear mixed model, reaction norms, thyroid hormones.

\footnotetext{
* Corresponding author; email: biyahs@utu.fi.

ORCIDs: Hsu, https://orcid.org/0000-0002-3799-0509; Verhagen, https://orcid .org/0000-0001-5588-1333; Gienapp, https://orcid.org/0000-0002-9368-8769; Visser, https://orcid.org/0000-0002-1456-1939; Ruuskanen, https://orcid.org/0000-0001-5582 $-9455$.

Am. Nat. 2019. Vol. 194, pp. E96-E108. (C) 2019 by The University of Chicago. 0003-0147/2019/19404-58962\$15.00. All rights reserved.

DOI: $10.1086 / 704738$
}

\section{Introduction}

Over the past decades, variation in maternal hormones transferred from mother to offspring has been found to profoundly influence the expression of morphological, physiological, and behavioral traits of the offspring (Love and Williams 2008; von Engelhardt and Groothuis 2011; Haussmann et al. 2012; Ruuskanen and Hsu 2018). Such effects can sometimes last long into adulthood (e.g., Hsu et al. 2016b). As a maternal trait, the variation of maternal hormones is determined mostly by internal and external factors that mothers have experienced. Researchers have therefore proposed that mothers may adaptively adjust hormone transfer in anticipation of the environment that the offspring will encounter (Marshall and Uller 2007).

Such "anticipatory maternal effects" (Marshall and Uller 2007) have several prerequisites, one of which is that mothers must possess sufficient flexibility to adjust the levels of transferred hormones. Previous studies have evaluated such phenotypic flexibility based mostly on the average change in maternal hormone levels between individuals from different groups (e.g., Hsu et al. 2016a; Ruuskanen et al. 2016a). A handful of studies also experimentally subjected the same females to different environments and evaluated the within-female plasticity of maternal hormone adjustment. For example, Kingma et al. (2009) found that the manipulation of male crown plumage ultraviolet reflectance during egg laying altered maternal androgen levels in the egg yolks of blue tits (Cyanistes caeruleus). However, regardless of the experimental design, previous studies have overlooked the individual variation in maternal hormone transfer. Individual plasticity in maternal hormone transfer, however, may be more relevant in the context of anticipatory maternal effects, as it may reveal how the plasticity is tailored to individual-specific intrinsic or extrinsic states and a different capacity to deliver anticipatory maternal effects across individuals. 
To properly assess individual variation in phenotypic plasticity requires repeatedly measuring the trait of the same individuals so that the between- and within-individual variation can be statistically partitioned (van de Pol and Wright 2009; Wilson et al. 2010; Dingemanse and Dochtermann 2013). Over the past decades, many studies of life-history and behavioral traits have adopted a mixed effects modeling approach to achieve such a task (Nussey et al. 2007; Dingemanse et al. 2009; Brommer 2013; Westneat et al. 2015). The increasingly available long-term data sets have also boosted the use of this method in estimating individual reaction norms of seasonal reproduction in response to global warming (e.g., Brommer et al. 2005, 2008; Nussey et al. 2005; Reed et al. 2006; Charmantier et al. 2008; Senapathi et al. 2011). Because physiological traits are known to harbor large between- and within-individual variation, the mixed model approach is therefore expected to fruitfully advance our understanding in physiological flexibility and natural selection on physiological traits (Williams 2008; Hau and Goymann 2015; Bonier and Martin 2016; Taff and Vitousek 2016). Nevertheless, the application of this method to physiological traits in wild animals started only recently, mostly in estimating the reaction norms of thermal sensitivity (e.g., Artacho et al. 2013; Careau et al. 2014), stress-induced responses (e.g., Lendvai et al. 2014, 2015; Fürtbauer et al. 2015), and the responses of plasma androgens and behavior to gonadotropin-releasing hormone $(\mathrm{GnRH})$ administration (e.g., Ambardar and Grindstaff 2017; Goymann and Dávila 2017). Studies that have assessed the variation in individual plasticity of maternal hormone transfer have only just started to emerge (e.g., Mentesana et al. 2019). This is somewhat surprising, as the variation of maternal hormone transfer has an essential role in determining the capacity of maternal hormones to mediate the effects on the offspring (i.e., transgenerational phenotypic plasticity) in the context of anticipatory maternal effects.

In this study, we used a unique data set, collected from a single great tit (Parus major) population over four consecutive years, to (i) quantify both the between- and the withinfemale variation of maternal thyroid hormones (THs) transferred into egg yolks and to (ii) estimate individual plasticity of egg THs across (1) environmental temperatures, (2) clutch initiation dates, and (3) egg laying order. We chose to focus on maternal THs because these maternal hormones have been overlooked until recently despite their well-established importance for embryonic development (Ruuskanen and Hsu 2018). Also, the variation of maternal THs at either between- or within-individual level or individual plasticity has not yet been characterized in any wild animals (Ruuskanen and Hsu 2018).

We hypothesized that there may be individual plasticity in maternal yolk THs over environmental temperatures and clutch initiation dates because maternal $\mathrm{TH}$ deposition is likely under physiological (and genetic) regulation during yolk formation (see the discussion in Hsu et al. 2016a; reviewed in Ruuskanen and Hsu 2018). Thyroid function is known to be influenced by changes in environmental temperature (McNabb and Darras 2015). In temperate birds, temperature change is also an important complementary cue aside from day lengths for the initiation of breeding (Schaper et al. 2012). Therefore, both environmental temperature and clutch initiation dates are possible covariates of yolk TH deposition, although the underlying mechanisms are unclear. In fact, a previous study indeed found that, at the population level, environmental temperature negatively associated with yolk thyroxine levels (T4, the main $\mathrm{TH}$ produced by the thyroid gland), although not with yolk triiodothyronine levels (T3, the most bioactive TH), in great tits (Ruuskanen et al. 2016d).

We also hypothesized that females may show individual plasticity in changes of yolk $\mathrm{TH}$ over the egg laying order, because laying order represents an important intrinsic within-clutch factor in birds. In many avian species, egg quality, reflected by the composition of eggs, usually systematically changes over the laying sequence (Williams 2012; Williams and Groothuis 2015). A previous study of great tits has indeed reported increasing T4 over the laying sequence at population level (Ruuskanen et al. 2016d).

All in all, we expected to see significant between-individual variation on yolk T3 but higher within-individual variation on yolk T4 given the previous findings that only yolk T3 and not T4 exhibited moderate heritability (Ruuskanen et al. 2016c). The direction of reaction norms across the chosen variables (see above) is difficult to predict a priori, however, because the previously found population-level reaction norm does not guarantee parallel plasticity at the individual level (Nussey et al. 2007).

\section{Material and Methods \\ Egg Sampling}

During 2013-2016, great tit females from a nest box breeding population in Bennekom, the Netherlands ( $51^{\circ} 59^{\prime} 53 \mathrm{~N}$, $5^{\circ} 40^{\prime} 35 \mathrm{E}$ ), were monitored during the breeding season. Eggs were collected on the day of laying and replaced with dummy eggs to ensure that no incubation of these eggs occurred. In this population, great tits raise one brood of nestlings in a breeding season but can lay a replacement clutch of eggs in case of early failure (Husby et al. 2009). Which eggs in a clutch were collected varied across years to accommodate other simultaneously ongoing experiments (Ruuskanen et al. 2016a, $2016 b$; Verhagen et al., forthcoming). In 2013, four or six eggs per clutch were collected (eggs 1, 2, 5, 6, 8, and 9 in the laying sequence), depending on clutch size. The yolks of two subsequent eggs were pooled for hormone assay $(1+2,5+6,8+$ 9) due to the need to also analyze yolk androgens for another 
study (Ruuskanen et al. 2016a). As a result, these data provided two or three data points per clutch in 2013 and were equivalent to two or three "functional" eggs for statistical analysis (see "Data Sets and Data Process"). In 2014, only one egg per clutch (egg 4) was collected. In 2015-2016, eggs 2 and 7 of the laying sequence were collected. From 2014-2016, the whole yolk of a collected egg was used for yolk TH analysis. The majority of the sampled eggs were from the first clutches ( $n=159$ clutches), and a small portion of the sampled eggs was from replacement clutches $(n=33$ clutches; fig. A1; figs. A1, A2 are available online). All eggs were stored at $-80^{\circ} \mathrm{C}$ from the day of collection until hormone analysis.

Over the course of this study, the spring temperatures exhibited a high variation among years, leading to substantial among-year difference in food availability and laying dates (see fig. A2). Females thus experienced a wide range of environmental conditions. Every breeding female was caught either during incubation or at the chick-rearing stage for ringing and identification. In total, we obtained 330 data points from 192 clutches laid by 119 females, with one to three data points per clutch (table A1; tables A1-A5 are available online).

\section{Hormone Analysis}

Yolk T3 and T4 were extracted and measured by radioimmunoassay (RIA) at Katholieke Universiteit Leuven, Belgium, every year within 5 months after the season ended. In a given year, all samples from an individual female were extracted in the same batch. We followed previously described protocols of TH extraction and RIA (Ruuskanen et al. 2016a). For eggs collected in 2013, yolks of two subsequent eggs in the same clutch $(1+2,5+6,8+9)$ were pooled and 400-500 mg of mixed yolk was used for TH extraction. For eggs collected in 2014-2016, the whole yolk (ca. $400 \mathrm{mg}$ ) of an egg was used for $\mathrm{TH}$ extraction. Yolks were first homogenized in methanol (three times the yolk weight), and 1,500-2,000 cpm of [ $\left.{ }^{131} \mathrm{I}\right] \mathrm{T} 3$ and $\left[{ }^{125} \mathrm{I}\right] \mathrm{T} 4$ were added as internal recovery tracers. Samples were then subjected to a series of extraction (chloroform in two times the volume of methanol), reextraction (2:1 mixture of chloroform and methanol), back extraction into an aqueous phase $\left(0.05 \% \mathrm{CaCl}_{2}\right)$, and reextraction $(3: 49: 48$ mixture of chloroform to methanol to $0.05 \% \mathrm{CaCl}_{2}$ ). The final aqueous phase was purified through Bio-Rad AG 1-X2 resin columns, eluted with $70 \%$ acetic acid, evaporated, and resuspended in RIA buffer. The recovery rates (mean $\pm \mathrm{SD}=$ $74.1 \% \pm 12.3 \%$ for $\mathrm{T} 3$ and $47.7 \% \pm 12.2 \%$ for $\mathrm{T} 4$ ) varied across extraction batches and years, and therefore the identity of extraction batches was included as a random effect and year was included as a fixed factor in the statistical analyses (see below). We used 20-TR45 and 20-TR40 antisera (Fitzgerald Industries, Acton, MA) in the RIA for T3 and T4, respectively. The T3 antiserum had $0.1 \%-0.5 \%$ cross-reactivity with T4, while the T4 antiserum cross-reactivity with T3 was 3.5\%. Intra-assay coefficients of variation (CVs) of the RIA were $2.2 \%$ and $2.8 \%$ for T3 and T4, respectively, while the interassay CVs were $9.6 \%$ and $11.0 \%$ for T3 and T4. In each year, all samples were measured in one single RIA assay. The detection limits of the RIA are $65 \mathrm{pg} / \mathrm{mL}$ for T3 and $777 \mathrm{pg} / \mathrm{mL}$ for T4. Because of the concentrating steps during the extraction procedure, this corresponds to $0.0065 \mathrm{pg} / \mathrm{mg}$ yolk for T3 and $0.0777 \mathrm{pg} / \mathrm{mg}$ yolk for T4. Our measurements were well above the detection limits $(\mathrm{T} 3$, mean $\pm \mathrm{SD}=0.139 \pm$ $0.136 \mathrm{pg} / \mathrm{mg}$ yolk, range: $0.005-0.585 \mathrm{pg} / \mathrm{mg}$; T4, mean \pm $\mathrm{SD}=1.390 \pm 1.235 \mathrm{pg} / \mathrm{mg}$ yolk, range: $0.041-6.602 \mathrm{pg} / \mathrm{mg})$, with only a few exceptions ( $n=1$ for T3 and $n=2$ for T4).

\section{Data Sets and Data Process}

Our data formed a nested structure containing four levels (fig. 1). The population was the highest level (level 0), in which we sampled 119 females in total (level 1). For each female, we sampled mostly one to three clutches (level 2). As explained above, mostly one to three "functional" eggs per clutch were assayed (level 3). Among the eggs collected and assayed for this study, we compiled two data sets. Data set 1 included all 330 eggs (table 1) and was used in the random intercept

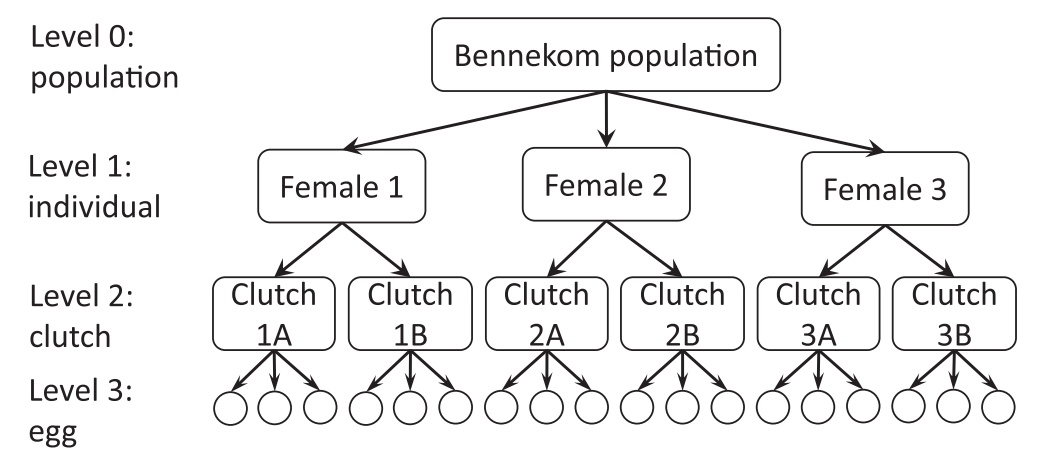

Figure 1: Schematic diagram of the nested data structure in this study. 
Table 1: Sample sizes at different levels in this study

\begin{tabular}{lccrrrr}
\hline & \multicolumn{2}{c}{ Data set 1 } & & \multicolumn{2}{c}{ Data set 2 } \\
\cline { 2 - 3 } & T3 & T4 & & T3 & T4 \\
\hline Level 1: individuals & 119 & 118 & & 24 & 23 \\
Level 2: clutches & 192 & 191 & & 53 & 51 \\
Level 3: "functional" eggs & 330 & 323 & & 111 & 107 \\
\hline
\end{tabular}

Note: The sample size for T4 is slightly lower than that for T3 due to insufficient amount of egg yolk for analysis and because we prioritized the assay of T3 over T4.

model and the female-level random slope models (see "Random Slope Mixed Models"). To further explore the withinindividual variation in maternal THs, we extracted a smaller subsample that contains multiple clutches laid by each female and also more than one egg per clutch (table 1, data set 2) for the multilevel random slope models (see "Random Slope Mixed Models"). Table 1 lists the sample size at each level, and table 2 lists the number of clutches sampled from each female in data sets 1 and 2 .

For the fixed effects of the models, which quantify the reaction norms of maternal THs at the population level, we considered the following candidate variables: year, egg laying order, environmental temperature during egg formation, clutch initiation date, clutch order (first or replacement clutch), and maternal age (1 year or older). Year was treated as a categorical variable with four levels (2013-2016). Egg laying order was represented by its proportional position in each clutch to account for variable clutch sizes, calculated following Ruuskanen et al. (2016d). For the eggs collected in 2013, the yolks from two subsequent eggs in the laying order were pooled for hormone analysis (see "Hormone Analysis"); we thus used the average laying order of these two eggs for calculation $(n=107)$. The average laying order was also used in case of uncertainty of laying order $(n=5)$. Temperature data were obtained from the closest Dutch meteorological station in De Bilt ( $\sim 10 \mathrm{~km}$ from our study site). Following Ruuskanen et al. $(2016 d)$, we calculated the average temperature from the hourly data during the 4 days prior to the laying of each egg, as the 4-day period represents rapid yolk formation (Badyaev et al. 2005). The environmental temperature during this period was previously found to negatively correlate with yolk T4 deposition (Ruuskanen et al. 2016d). The following procedures were then used to separate the withinand between-clutch effects of temperature: we first calculated clutch mean temperatures by averaging the calculated environmental temperatures of all eggs in a clutch. We then calculated a deviation from the clutch mean temperature for each egg by subtracting the clutch mean temperature from the calculated environmental temperature of an egg. Clutch mean temperature was also centered to the grand mean. The clutch initiation dates were represented by the laying date of the first egg of a clutch, and hence every egg from the same clutch had the same value for this variable. We then mean centered the clutch initiation date by year so that it represents how early or late a clutch was laid in each year. Clutch order was a two-level categorical variable, for which the replacement clutches were judged by the actual records (clutches from the same female have been collected in the same season) or late laying dates (clutch lay date $>30$ days from the first clutch of the season; van Noordwijk et al. 1995; fig. A1).

\section{Random Slope Mixed Models}

While the population-level effects were modeled by the fixed factors, the female- and clutch-level effects needed to be modeled as random effects at the corresponding level. We considered only linear reaction norms because our data were not sufficient to reliably model nonlinear random effects. For example, a second-order polynomial reaction norm requires at least three data points per female-namely, 357 eggs for our 119 females - but we had only 330 eggs. Even restricting the data set to the females from which at least three eggs were sampled (214 eggs from 52 females for T3 and 209 eggs from

Table 2: Number of clutches sampled from each female in data sets 1 and 2

\begin{tabular}{|c|c|c|c|c|c|c|c|c|c|c|}
\hline & & & T3 & & & & & $\mathrm{T} 4$ & & \\
\hline \multicolumn{11}{|l|}{ Data set 1 : } \\
\hline No. clutches per female & 1 & 2 & 3 & 4 & 6 & 1 & 2 & 3 & 4 & 6 \\
\hline No. clutches & 70 & 60 & 48 & 8 & 6 & 69 & 60 & 48 & 8 & 6 \\
\hline No. females & 70 & 30 & 16 & 2 & 1 & 69 & 30 & 16 & 2 & 1 \\
\hline \multicolumn{11}{|l|}{ Data set 2 : } \\
\hline No. clutches per female & 1 & 2 & 3 & 4 & 6 & 1 & 2 & 3 & 4 & 6 \\
\hline No. clutches & 0 & 38 & 15 & 0 & 0 & 0 & 36 & 15 & 0 & 0 \\
\hline No. females & 0 & 19 & 5 & 0 & 0 & 0 & 18 & 5 & 0 & 0 \\
\hline
\end{tabular}

Note: The sample size for T4 is slightly lower than that for T3 due to insufficient amount of egg yolk for analysis and because we prioritized the assay of T3 over T4. In data set 2, only those clutches from which we sampled more than one egg (see table A1, available online) and those females from which we sampled more than one clutch were included. Data set 1 was used in the analysis of the random intercept model and the female-level random slope models, whereas data set 2 was used in the analysis of the multilevel random slope models. 
51 females for T4) did not give reliable estimates (see "FemaleLevel Random Slope Model").

A linear reaction norm consists of two parameters: the intercept that describes the "elevation" of the reaction norm, representing the average phenotypic expression, and the slope that represents the extent of phenotypic change over the environmental gradient (i.e., the plasticity; Nussey et al. 2007; Brommer 2013; Westneat et al. 2015). Because the fixed effects included in the models will influence the residual variance (Araya-Ajoy et al. 2015), we first ran a random-intercept-only model with data set 1 in search of a most simplified structure of fixed effects. In this random intercept model, clutch ID, female ID, and the batch of hormone extraction were included as random intercepts to account for the corresponding nonindependence of data points. All the abovementioned candidate variables were included as fixed factors initially (table A2), and nonsignificant variables were subsequently removed through a backward elimination procedure. The final model retained only year and egg laying order, which were both statistically significant (table A3). Quadratic effects of environmental temperature and egg laying order were tested using the final random intercept model but turned out nonsignificant. Therefore, only year and egg laying order were considered the essential fixed effects in the further multilevel random slope models.

Among all the candidate variables, laying dates showed substantial correlation with clutch mean temperature $(r=$ $0.38)$ and clutch order $(r=0.84)$. The former correlation did not lead to strong collinearity (variance inflation factor [VIF] $<1.5$ in all cases). The latter correlation raised a warning, but both factors exhibited nonsignificant populationlevel effects on maternal TH deposition, and neither was included in the final model. In the final random-intercept-only model and all further random slope models, no clear multicollinearity was detected (all VIFs <1.5).

Multilevel Random Slope Model. The multilevel random slope models were used to partition the between-individual and between-clutch variation of maternal yolk TH deposition, so the individual- and clutch-level effects can be estimated. Random slope models always contain necessary random intercept terms. The model formula is expressed as

$$
\begin{aligned}
y_{i j k}= & \mu_{F}+\beta_{F} E_{i j k}+\left(\beta_{0}+\text { Bat }_{0 l}+\text { Ind }_{0 k}+C l u_{0 j k}\right) \\
& +\left(\beta_{1}+I_{1 n d}+C l u_{1 j k}\right) E_{i j k}+\varepsilon_{0 i j k},
\end{aligned}
$$

where $y_{i j k}$ represents yolk THs measured at egg $i$ from clutch $j$ laid by individual female $k$. The parameter $E_{i j k}$ represents the variables of interest that may influence yolk TH deposition. In these models, we tested two variables that were measured for each egg and hence showed variation within clutches as random slopes-environmental temperature during yolk formation and egg laying order. Fixed ef- fects were represented by $\mu_{F}$ and $\beta_{F}$. The fixed effects included year and egg laying order, as suggested by the randomintercept-only model, and also the random slope term if not yet already included. Separate models were fitted when different random slope terms were used. With this model, we were able to estimate the reaction norm elevation $\left(\beta_{0}+I n d_{0 k}\right)$ and slope $\left(\beta_{1}+\right.$ Ind $\left._{1 k}\right)$ as well as the deviation of each clutch from each individual female's mean reaction norm elevation $\left(C l u_{0 j k}\right)$ and slope $\left(C l u_{1 j k}\right)$. The batch of hormone extraction was included as an additional random intercept $\left(B a t_{0 l}\right)$ to account for the potential nonindependence within each batch. The error term $\varepsilon_{0 i j k}$ represented the unexplained within-clutch variation of maternal $\mathrm{TH}$ deposition.

The multilevel random slope model requires repeated measures in each clutch and multiple clutches from each female, and therefore we were compelled to use the smaller data set 2 (tables 1,2). We therefore fitted the second set of random slope models to focus on between-female variation of yolk TH deposition with the much larger sample size in data set 1 .

Female-Level Random Slope Model. This set of female-level random slope models is expressed as

$$
\begin{aligned}
y_{i j k}= & \mu_{F}+\beta_{F} E_{i j k}+\left(\beta_{0}+B a t_{0 l}+\operatorname{Ind}_{0 k}+C l u_{0 j k}\right) \\
& +\left(\beta_{1}+\operatorname{Ind}_{1 k}\right) E_{i j k}+\varepsilon_{0 i j k} .
\end{aligned}
$$

In this set of models, data of eggs laid in different clutches by the same female were pooled to estimate a female-specific slope. Nevertheless, clutch ID was still included as a random intercept $\left(\mathrm{Clu}_{0 j k}\right)$ to control for the nonindependence among the eggs from the same clutch. The variables tested as random slope terms were the clutch mean environmental temperature during yolk formation, yearly centered clutch initiation date, and egg laying order. To avoid confusion with the above models containing both the female- and the clutchlevel random slopes, these models were called female-level random slope models, despite the fact that it still contained two levels (population and female levels).

We exploratively tested the possibility of quadratic random slopes using a second-order polynomial formula of environmental temperature, clutch initiation date, and egg laying order as random slopes. These quadratic random slopes all turned out to be nonsignificant with warnings on convergence. We thus cannot be certain of the reliability of these explorative tests and reported and discussed only the linear reaction norms in this article.

All models were fitted with $\mathrm{R}$ package lme4 (Bates et al. 2015) in $R$ version 3.3.1 ( $R$ Core Team 2016). The significance of fixed effects was tested by model comparisons with a Kenward-Roger approximation (R package pbkrtest; Halekoh and Højsgaard 2014). The significance of random intercepts 
and slopes were determined by model comparisons with loglikelihood ratio tests. The data included in this study as well as $\mathrm{R}$ codes of our models have been deposited in the Dryad Digital Repository (https://doi.org/10.5061/dryad.kp524f5; Hsu et al. 2019b).

\section{Results}

\section{Multilevel Random Slope Models}

For yolk T3, our multilevel random slope models did not detect statistically significant random slopes of temperature or egg laying order across clutches or individuals (table 3 ). These suggested no significant between-individual and betweenclutch variation in the plasticity of yolk T3. For yolk T4, however, the model including egg laying order, as the random slope at the clutch level performed significantly better than the model without it (table 3 ), suggesting significant variation of slopes over the laying order across clutches (fig. 2). The random slope of T4 against temperature during egg formation did not show significant variation (table 3 ), suggesting little evidence of between-clutch difference in the plasticity of $\mathrm{TH}$ deposition across temperature. These results, how- ever, should be interpreted with caution because of the lower sample size.

\section{Female-Level Random Slope Models}

The larger sample size in data set 1 allowed us to more robustly explore the between- and within-female variation of maternal TH deposition. This set of random slope models interestingly suggested that the random intercepts were significant for yolk T3 but not T4 (table 4). The significance of the random intercepts suggested a large between-female variation of the average yolk T3 relative to within-female variation (fig. 3). No significant random slopes were detected for either yolk T3 or T4, independent of which variable was tested (table 4). This suggested small between-female variation relative to withinfemale variation in the plasticity of maternal $\mathrm{TH}$ deposition.

\section{Population-Level Effects}

The population-level effects from most of the random slope models showed consistent results with the random intercept model (see "Material and Methods"); namely, only the year and the egg laying order effect were statistically significant

Table 3: Multilevel random slope models of yolk T3 and T4

\begin{tabular}{|c|c|c|c|c|c|c|c|c|}
\hline Model & df & AIC & Log likelihood & Deviance & Test & $\chi^{2}$ & $\Delta \mathrm{df}$ & $P$ \\
\hline & \multicolumn{8}{|c|}{ Yolk T3 (111 eggs, 53 clutches, 24 females) } \\
\hline & \multicolumn{8}{|c|}{$X_{1}=$ deviation from clutch mean temperature for each egg; $X_{2}=$ mean-centered clutch mean temperature } \\
\hline 1 & 14 & 137.28 & -54.639 & 109.28 & & & & \\
\hline 2 & 12 & 134.00 & -54.999 & 110.00 & 1 vs. 2 & .720 & 2 & .698 \\
\hline \multirow[t]{2}{*}{3} & 10 & 133.09 & -56.544 & 113.09 & 2 vs. 3 & 3.090 & 2 & .213 \\
\hline & \multicolumn{8}{|c|}{$X_{1}=X_{2}=$ proportional egg laying order } \\
\hline 1 & 12 & 136.12 & -56.062 & 112.12 & & & & \\
\hline 2 & 10 & 133.57 & -56.784 & 113.57 & 1 vs. 2 & 1.444 & 2 & .486 \\
\hline \multirow[t]{3}{*}{3} & 8 & 129.57 & -56.784 & 113.57 & 2 vs. 3 & $<.001$ & 2 & $>.999$ \\
\hline & \multicolumn{8}{|c|}{ Yolk T4 (323 eggs, 191 clutches, 118 females) } \\
\hline & \multicolumn{8}{|c|}{$X_{1}=$ deviation from clutch mean temperature; $X_{2}=$ mean-centered clutch mean temperature } \\
\hline 1 & 14 & 187.07 & -79.504 & 159.01 & & & & \\
\hline 2 & 12 & 183.40 & -79.698 & 159.40 & 1 vs. 2 & .389 & 2 & .823 \\
\hline \multirow[t]{2}{*}{3} & 10 & 179.62 & -79.808 & 159.62 & 2 vs. 3 & .220 & 2 & .896 \\
\hline & \multicolumn{8}{|c|}{$X_{1}=X_{2}=$ proportional egg laying order } \\
\hline 1 & 12 & 168.76 & -72.380 & 144.76 & & & & \\
\hline 2 & 10 & 180.61 & -80.303 & 160.61 & 1 vs. 2 & 15.845 & 2 & $<.001$ \\
\hline 3 & 8 & 177.07 & -80.536 & 161.07 & 2 vs. 3 & .467 & 2 & .792 \\
\hline
\end{tabular}

Note: Model comparisons were performed by changing the random effects structure in the following order: $1=\left(X_{1} \mid\right.$ clutch ID $)+\left(X_{2} \mid\right.$ female ID); $2=$ $(1 \mid$ clutch ID $)+\left(X_{2} \mid\right.$ female ID $) ; 3=(1 \mid$ clutch ID $)+(1 \mid$ female ID $)$, where $X_{1}$ and $X_{2}$ represent the random slope term that interacts with clutch ID and female ID, respectively. When the random slope term was dropped, 1 was added to represent the intercept. The comparison between models 1 and 2 determines whether the variation of the random slope $X_{1}$ at the clutch level is significant; the comparison between models 2 and 3 determines whether the variation of the random slope $X_{2}$ at the female level is significant. The significance is determined by log-likelihood ratio tests. Boldfacing indicates statistical significance. AIC = Akaike information criterion. 


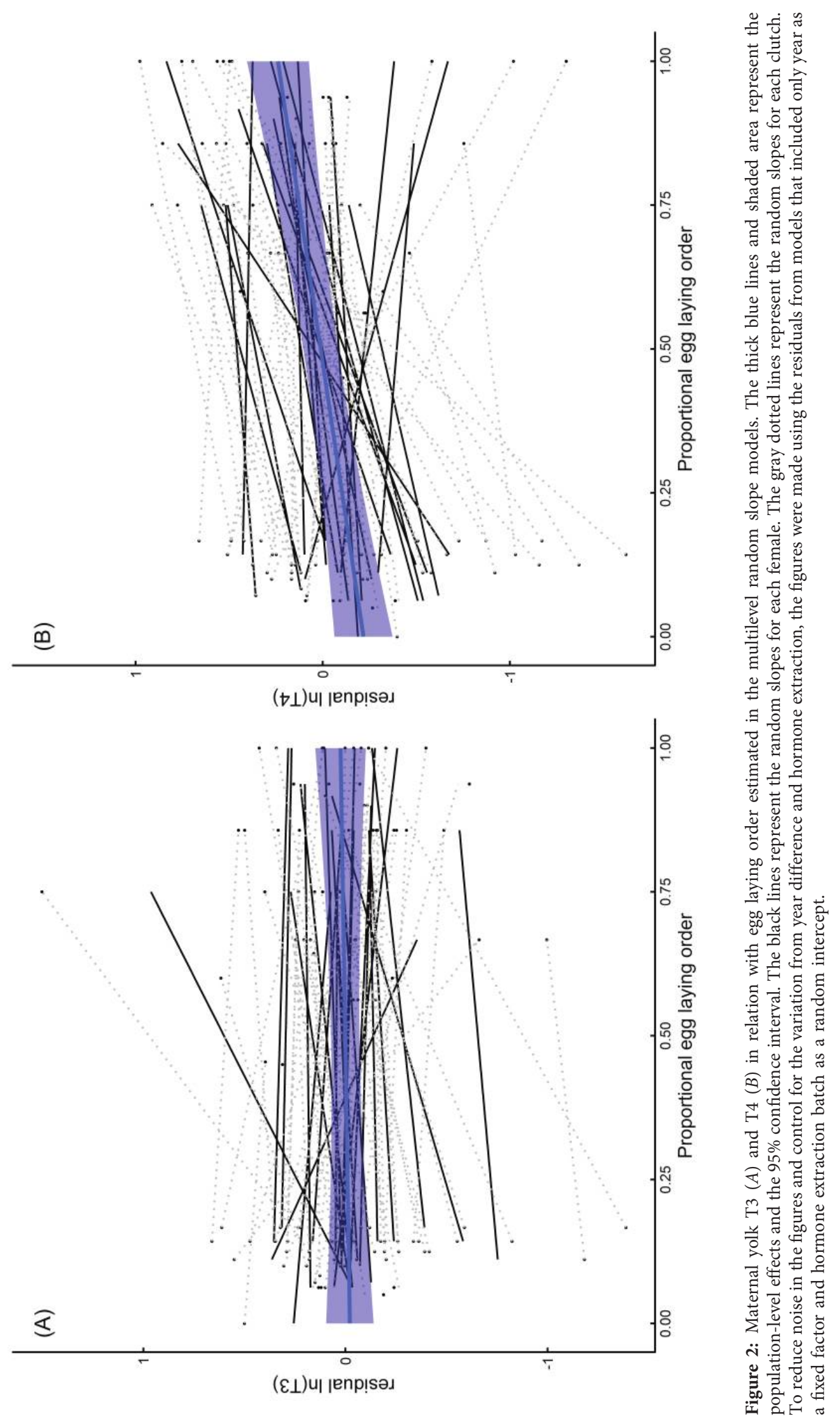

E102

This content downloaded from 134.058.253.056 on October 02, 2019 07:19:47 AM 
Table 4: Female-level random slope models of yolk T3 and T4

\begin{tabular}{|c|c|c|c|c|c|c|c|c|}
\hline Model & df & AIC & Log likelihood & Deviance & Test & $\chi^{2}$ & $\Delta \mathrm{df}$ & $P$ \\
\hline & \multicolumn{8}{|c|}{ Yolk T3 (330 eggs, 192 clutches, 119 females) } \\
\hline & \multicolumn{8}{|c|}{$X=$ mean-centered clutch mean temperature } \\
\hline 1 & 13 & 364.72 & -169.36 & 338.72 & & & & \\
\hline 2 & 11 & 362.75 & -170.37 & 340.75 & 1 vs. 2 & 2.025 & 2 & .363 \\
\hline \multirow[t]{2}{*}{3} & 10 & 370.15 & -175.07 & 350.15 & 2 vs. 3 & 9.367 & 1 & .002 \\
\hline & \multicolumn{8}{|c|}{$X=$ yearly centered clutch lay date } \\
\hline 1 & 12 & 362.81 & -169.41 & 338.81 & & & & \\
\hline 2 & 10 & 359.02 & -169.51 & 339.02 & 1 vs. 2 & .205 & 2 & .903 \\
\hline \multirow[t]{2}{*}{3} & 9 & 367.57 & -174.79 & 349.57 & 2 vs. 3 & 10.554 & 1 & .001 \\
\hline & \multicolumn{8}{|c|}{$X=$ proportional egg laying order } \\
\hline 1 & 11 & 360.05 & -169.02 & 338.05 & & & & \\
\hline 2 & 9 & 359.84 & -170.92 & 341.84 & 1 vs. 2 & 3.786 & 2 & .151 \\
\hline \multirow[t]{3}{*}{3} & 8 & 367.52 & -175.76 & 351.52 & 2 vs. 3 & 9.683 & 1 & .002 \\
\hline & \multicolumn{8}{|c|}{ Yolk T4 (323 eggs, 191 clutches, 118 females) } \\
\hline & \multicolumn{8}{|c|}{$X=$ mean-centered clutch mean temperature } \\
\hline 1 & 13 & 423.38 & -198.69 & 397.38 & & & & \\
\hline 2 & 11 & 424.64 & -201.32 & 402.64 & 1 vs. 2 & 5.263 & 2 & .072 \\
\hline \multirow[t]{2}{*}{3} & 10 & 423.22 & -201.61 & 403.22 & 2 vs. 3 & .580 & 1 & .446 \\
\hline & \multicolumn{8}{|c|}{$X=$ yearly centered laying date } \\
\hline 1 & 12 & 429.44 & -202.72 & 405.44 & & & & \\
\hline 2 & 10 & 425.62 & -202.81 & 405.62 & 1 vs. 2 & .179 & 2 & .915 \\
\hline \multirow[t]{2}{*}{3} & 9 & 424.05 & -203.03 & 406.05 & 2 vs. 3 & .430 & 1 & .512 \\
\hline & \multicolumn{8}{|c|}{$X=$ proportional egg laying order } \\
\hline 1 & 11 & 426.34 & -202.17 & 404.34 & & & & \\
\hline 2 & 9 & 423.75 & -202.88 & 405.75 & 1 vs. 2 & 1.411 & 2 & .494 \\
\hline 3 & 8 & 422.25 & -203.13 & 406.25 & 2 vs. 3 & .499 & 1 & .480 \\
\hline
\end{tabular}

Note: Model comparisons were performed in the following order: $1=(1 \mid$ clutch ID $)+(X \mid$ female ID $) ; 2=(1 \mid$ clutch ID $)+(1 \mid$ female ID $) ; 3=(1 \mid$ clutch ID $)$, where $X$ represents the random slope term that interacts with female ID. The random intercept of clutch ID was still included to account for the nonindependence among eggs from the same clutches. The comparison between models 1 and 2 determines whether the variation of the random slope $X$ at the female level is significant; the comparison between models 2 and 3 determines whether the variation of the random intercept of female ID is significant. The significance is determined by loglikelihood ratio tests. Boldfacing indicates statistical significance. AIC = Akaike information criterion.

(see tables A3-A5). These results indicated that at the population level, both yolk T3 and T4 varied across years and both increased over the laying sequence (see thick blue lines in figs. 2, 3; tables A3-A5). The only exception was the multilevel random slope models for yolk T3, in which the populationlevel effects of egg laying order were not significant (table A4). This was very likely due to insufficient statistical power in that model because of the much smaller sample size $(n=111$ eggs from 53 clutches and 24 females; table A4).

\section{Discussion}

Making use of a unique data set of maternal yolk THs from a wild population of great tits across 4 years, we were able to partition the variation at between-individual, between-clutch, and within-clutch levels and examine the reaction norms at population, female, and clutch levels. Yolk T3 shows significant between-individual variation in the average concentrations (i.e., random intercepts), whereas yolk T4 shows large variation in the slopes over the laying sequence between clutches of individual females (i.e., random slopes). This suggests that the two THs - T3 and T4-deposited in egg yolks by females exhibit different patterns of variation and that females are capable of adjusting the slope for each clutch of eggs.

\section{Between-Individual Variation of Maternal THs}

In the female-level random slope models, we found that regardless of which random slope term (temperature, clutch 


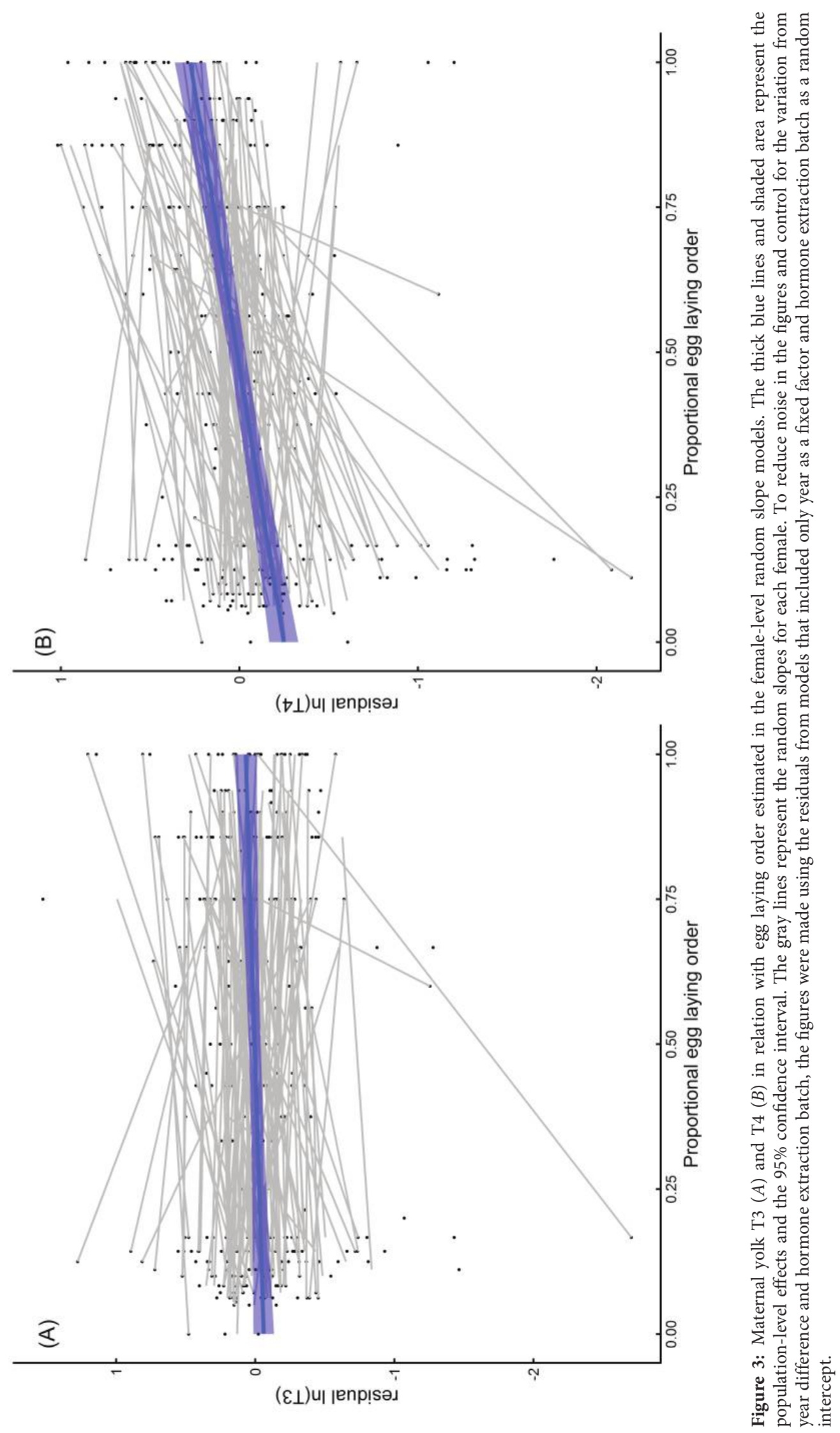

E104

This content downloaded from 134.058.253.056 on October 02, 2019 07:19:47 AM 
initiation date, egg laying order) was tested, yolk T3 always showed significant between-individual variation on the intercept but not on the slope. This indicates significant individual differences in the average levels of yolk T3 (variable elevations of the gray lines in fig. $3 A$ ) but no individual differences in the plasticity of T3 deposition across environmental temperature, clutch initiation dates, or egg laying order. As T3 is the most biologically active form of THs, perhaps its plasticity is under a strict control to avoid potential detrimental effects due to a large magnitude of fluctuation. This result is in line with a recent study of eastern bluebirds (Sialia sialis) that found individual differences in plasma testosterone levels only on the intercept (i.e., the average level) but not on the response to GnRH (Ambardar and Grindstaff 2017). In contrast with T3, significant between-individual variation was not detected for yolk T4. This disparity suggests that T4 is more susceptible to environmental fluctuation while T3 is under a stricter physiological and genetic control, in line with the finding that only yolk T3 exhibited moderate heritability $\left(h^{2}=0.25\right)$ and not T4 $\left(h^{2}=3.32 \mathrm{e}-07\right.$; Ruuskanen et al. 2016c). A similar scenario in yolk androgens was also previously reported: the biologically active testosterone also had higher heritability $\left(h^{2}=0.75\right)$ than its prohormone androstenedione $\left(h^{2}=-0.20\right.$; Tschirren et al. 2009; but see Okuliarova et al. 2011; Ruuskanen et al. 2016c). Testosterone also showed high within-female consistency (Tobler et al. 2007; but see Müller et al. 2012). Together, these results could suggest that the disparate patterns of variation may be a shared feature representing different roles played by an active hormone and its prohormone (e.g., Gomez et al. 1997). However, we also have to keep in mind that apart from being a prohormone for T3, T4 can also have functions of its own. For instance, some studies reported that only T4 treatment and not T3 could restore the normal TH function after thyroidectomy or TH inhibition by methimazole (Pant and Chandola-Saklani 1995; Pérez et al. 2018). T4 is also known to have stimulatory effects on angiogenesis and on the cytoskeleton via nongenomic pathways (Cheng et al. 2010).

Statistically significant individual plasticity (i.e., random slopes at the female level) against environmental temperature, clutch initiation dates, and egg laying order was not found for yolk T3 or T4, in contrast to our predictions. This result may be interpreted as small between-individual variation in slopes, which usually manifests itself as parallel reaction norms across individuals (e.g., Charmantier et al. 2008; Senapathi et al. 2011). This does not seem to be the case, however, as in our data the individual slopes apparently varied to a great extent (gray lines in fig. 3). A more likely alternative explanation is to attribute the lack of individual difference in plasticity to the large within-individual variation, reducing the statistical power to detect significant individual differences. This explanation appears to be more congruent with our data, as significant between-clutch variation on the slopes of yolk T4 across egg laying order within females was indeed detected (gray dotted lines in fig. 2B).

The large within-individual variation may also result from other reasons (Westneat et al. 2015), for example, if the yolk THs had nonlinear reaction norms. Nonlinear reaction norms have been reported in wild systems at the population level (e.g., Westneat et al. 2011; Goymann and Dávila 2017), but in our case the quadratic effects of temperature and egg laying order at the population level (i.e., fixed effects) were not statistically significant (see "Material and Methods"). At the individual level, we did not have sufficient data to reliably model it, and so far very little about nonlinear reaction norms has been explored. This is likely because the required sample size to model individual reaction norms is generally very large (Martin et al. 2011; van de Pol 2012). Theoretical simulation studies actually suggested a minimal sample size of 200 as a rule of thumb (Martin et al. 2011), but even larger might be needed to reach an ideal statistical power for linear reaction norms (van de Pol 2012), let alone modeling nonlinear reaction norms. For the future, including regular egg sampling and yolk hormone measurements as part of the long-term monitoring may be a solution.

\section{Within-Individual Variation of Maternal THs}

As previously stated, the large within-individual variation in yolk T3 and T4 is the more likely explanation for the lack of significant individual plasticity. For the biological significance of within-individual phenotypic variation, we unfortunately still know very little (Westneat et al. 2015). Very often, withinindividual phenotypic variation is treated as random, unexplained residual variation. This is partly because many sources of within-individual variation are nonbiological, such as measurement errors. Another reason is that there is usually no clearly identifiable cluster within individuals to help further partition the within-individual variance. In our present study, however, we were able to further break down the withinindividual variation to between- and within-clutch variation. Interestingly, our results indicated significant between-clutch variation of yolk T4 in the slopes over the laying sequence. This suggested that a female has the ability to flexibly alter the pattern of T4 deposition over the laying sequence across clutches (dotted lines in fig. $2 B$ ). The large between-clutch variation in the plasticity of yolk T4 also contributed to the large within-individual variation, partly explaining the lack of significant between-individual variation of yolk T4.

Aside from the between-clutch variation, there is still considerable within-individual variation of both yolk THs that is unexplained by our data. The pooling of yolks for the eggs collected in 2013 may have also contributed to some withinindividual variation. Moreover, the data of environmental temperature might not be what the birds actually perceivedthat is, there may be errors in animals' assessment of the 
environment, which may have contributed to the unexplained variation ("organismal error" in Westneat et al. 2015). Animals may also deploy behavioral strategies to avoid or mitigate inclement weather, such as searching for covers, which may have influenced their physiological responses to environmental fluctuation. As great tits mostly lay only one clutch in a breeding season, the large within-individual variation may also reflect year-related differences.

The flexibility of maternal TH deposition within individuals has important implications for the function of maternal yolk THs in birds. Traditionally, maternal yolk hormones in birds have been hypothesized to influence the dynamics of sibling competition (Groothuis et al. 2005). This hypothesis is proposed based on the findings that in many species of birds, yolk androgens increase over the laying sequence and higher yolk androgens enhance growth and begging behavior (Schwabl 1993; Groothuis et al. 2005). Higher androgens in the later-laid eggs may thus help the younger siblings survive (Schwabl 1993; Groothuis et al. 2005). Like maternal androgens, maternal THs might also influence sibling competition by enhancing nestling growth (Hsu et al. 2019a; but see Ruuskanen et al. 2016b; Hsu et al. 2017). In this case, differential T4 deposition over the laying sequence might increase offspring or maternal fitness or both, and selection would therefore preserve the flexibility of yolk T4 deposition. Experiments with a within-female design — namely, subjecting the same females to different states of an internal or external factor-will be valuable in identifying the determinants of the within-clutch pattern of yolk THs. Alternatively, the between-clutch variation of yolk T4 over the laying sequence could simply reflect the T4 fluctuation in the maternal blood circulation. As the mechanisms of maternal yolk TH deposition are still unknown (Ruuskanen and Hsu 2018) and we do not have blood TH data from the female great tits whose eggs had been sampled, we cannot rule out this possibility.

Despite the between-clutch variation in the plasticity of T4, we did not detect significant between-clutch variation on the average yolk T4. One explanation for this is again because of a large within-clutch variation, which begs a question: can female birds alter yolk T4 deposition to each egg in response to a short-term internal or external factor? A previous experiment in blue tits showed that a female could alter yolk androgens in the subsequent eggs after manipulation of her partner's plumage coloration (Kingma et al. 2009). Therefore, such a flexibility might be possible but still requires further testing for yolk THs (e.g., Ruuskanen et al. 2016a). Within-clutch variation of maternal THs itself may be inherently beneficial for the mothers, as it increases the phenotypic variation of the offspring and thus may function as a bet-hedging strategy (Crean and Marshall 2009; Shama 2015) to a rapidly changing and unpredictable environment.

\section{Variation and Plasticity of Yolk THs: Passive Outcome or Adaptive Response?}

Currently, the physiological mechanism of TH deposition in the egg yolks is still unknown, but there are many elements that likely play important roles, such as transmembrane transporters and deiodinase enzymes that metabolize THs. Individual differences in maternal expression of these elements may contribute to the individual difference of average yolk T3 as well as the between-clutch flexibility of T4. These transporters and deiodinases are also expressed at the yolk sac membrane in the developing embryo (Too et al. 2017). As these regulators have different affinities for T3 and $\mathrm{T} 4$, the complex interplay among them will serve to ensure that the embryonic tissues get the right amount of THs. Future work is therefore still needed to identify the driving factors underlying the between-clutch flexibility of yolk T4 from a mechanistic point of view.

Experiments from a functional point of view can also help determine whether adjusting yolk THs would increase either maternal or offspring fitness or both. So far, the limited experimental studies reported mixed results. In great tits, elevated yolk THs (mixture of T3 and T4) within the natural range enhanced growth in male nestlings but reduced it in female nestlings (Ruuskanen et al. 2016b). In contrast, similar experimental elevation of yolk THs was found decreasing nestling body mass in rock pigeons (Columba livia) and enhancing nestling growth in collared flycatchers (Ficedula albicollis) independently of sex (Hsu et al. 2017, 2019a). Interestingly, our unpublished data on domesticated Japanese quails (Coturnix japonica domesticus) showed that yolk T4 alone, and not T3, could enhance hatching success by ca. 20\% (T. Sarraude, B.-Y. Hsu, and S. Ruuskanen, unpublished data). If such a function holds true in wild birds, different slopes of T4 over the laying sequence might influence sibling competition by influencing how many nestlings would successfully hatch.

In conclusion, our unique 4-year data set provided statistical evidence that the average maternal T3 levels deposited in the egg yolks differed across individuals, while maternal yolk T4 showed large flexibility across clutches within females. The disparate patterns of variation between maternal T3 and T4 may at least partially point toward different roles in the endocrine systems. Whether the individual difference and within-individual flexibility are adaptive, however, remains to be investigated.

\section{Acknowledgments}

We greatly thank Jon Brommer for statistical consultation in the early stage of statistical analysis and numerous students and helpers for valuable help in the field work. The study is funded by the Academy of Finland. 


\section{Literature Cited}

Ambardar, M., and J. L. Grindstaff. 2017. Pre-GnRH and GnRHinduced testosterone levels do no vary across behavioral contexts: a role for individual variation. General and Comparative Endocrinology 246:51-62.

Araya-Ajoy, Y. G., K. J. Mathot, and N. J. Dingemanse. 2015. An approach to estimate short-term, long-term and reaction norm repeatability. Methods in Ecology and Evolution 6:1462-1473.

Artacho, P., I. Jouanneau, and J.-F. Le Galliard. 2013. Interindividual variation in thermal sensitivity of maximal sprint speed, thermal behavior, and resting metabolic rate in a lizard. Physiological and Biochemical Zoology 86:458-469.

Badyaev, A. V., H. Schwabl, R. L. Young, R. A. Duckworth, K. J. Navara, and A. F. Parlow. 2005. Adaptive sex differences in growth of preovulation oocytes in a passerine bird. Proceedings of the Roval Society B 272:2165-2172.

Bates, D., M. Maechler, B. Bolker, and S. Walker. 2015. Fitting linear mixed-effects models using lme4. Lournal of Statistical Software 67:1-48.

Bonier, F., and P. R. Martin. 2016. How can we estimate natural selection on endocrine traits? lessons from evolutionary biology. Proceedings of the Roval Societv B 283:20161887.

Brommer, J. E. 2013. Phenotypic plasticity of labile traits in the wild. Current Zoology 59:485-505.

Brommer, J. E., J. Merilä, B. C. Sheldon, and L. Gustafsson. 2005. Natural selection and genetic variation for reproductive reaction norms in a wild bird population. Evolution 59:1362-1371.

Brommer, J. E., K. Rattiste, and A. J. Wilson. 2008. Exploring plasticity in the wild: laying date-temperature reaction norms in the common gull Larus canus. Proceedings of the Roval Societv B 275: 687-693.

Careau, V., M. E. Gifford, and P. A. Biro. 2014. Individual (co)variation in thermal reaction norms of standard and maximal metabolic rates in wild-caught slimy salamanders. Functional Ecology 28:1175-1186.

Charmantier, A., R. H. McCleery, L. R. Cole, C. Perrins, L. E. B. Kruuk, and B. C. Sheldon. 2008. Adaptive phenotypic plasticity in response to climate change in a wild bird population. Science 320:800-803.

Cheng, S.-Y., J. L. Leonard, and P. J. Davis. 2010. Molecular aspects of thyroid hormone actions. Endocrine Reviews 31:139-170.

Crean, A. J., and D. J. Marshall. 2009. Coping with environmental uncertainty: dynamic bet hedging as a maternal effect. Philosophical Transactions of the Roval Society B 364:1087-1096.

Dingemanse, N. J., and N. A. Dochtermann. 2013. Quantifying individual variation in behaviour: mixed-effect modelling approaches. Journal of Animal Ecology 82:39-54.

Dingemanse, N. J., A. J. N. Kazem, D. Réale, and J. Wright. 2009. Behavioural reaction norms: animal personality meets individual plasticity. Trends in Ecology and Evolution 25:81-89.

Fürtbauer, I., A. Pond, M. Heistermann, and A. J. King. 2015. Personality, plasticity and predation: linking endocrine and behavioural reaction norms in stickleback fish. Functional Ecology 29:931-940.

Gomez, J. M., T. Boujard, G. Boeuf, A. Solari, and P.-Y. Le Bail. 1997. Individual diurnal plasma profiles of thyroid hormones in rainbow trout (Oncorhynchus mykiss) in relation to cortisol, growth hormone, and growth rate. General and Comparative Endocrinology 107:74-83.

Goymann, W., and P. F. Dávila. 2017. Acute peaks of testosterone suppress paternal care: evidence from individual hormonal reaction norms. Proceedings of the Roval Society B 284:20170632.
Groothuis, T. G. G., W. Müller, N. von Engelhardt, C. Carere, and C. Eising. 2005. Maternal hormones as a tool to adjust offspring phenotype in avian species. Neuroscience and Biobehavioral Reviews 29:329-352.

Halekoh, U., and S. Højsgaard. 2014. A Kenward-Roger approximation and parametric bootstrap methods for tests in linear mixed models the R package pbkrtest. Iournal of Statistical Software 59:1-30.

Hau, M., and W. Goymann. 2015. Endocrine mechanisms, behavioral phenotypes and plasticity: known relationships and open questions. Frontiers in Zoology 12:S7.

Haussmann, M. F., A. S. Longenecker, N. M. Marchetto, S. A. Juliano, and R. A. Bowden. 2012. Embryonic exposure to corticosterone modifies the juvenile stress response, oxidative stress and telomere length. Proceedings of the Roval Societv B 279:1447-1456.

Hsu, B.-Y., C. Dijkstra, V. M. Darras, B. de Vries, and T. G. G. Groothuis. 2016. Maternal adjustment or constraint: differential effects of food availability on maternal deposition of macronutrients, steroids and thyroid hormones in rock pigeon eggs. Ecology and Evolution 6:397-411.

2017. Maternal thyroid hormones enhance hatching success but decrease nestling body mass in the rock pigeon (Columba livia). General and Comparative Endocrinology 240:174-181.

Hsu, B.-Y., C. Dijkstra, and T. G. G. Groothuis. 2016b. No escape from mother's will: effects of maternal testosterone on offspring reproductive behaviour far into adulthood. Animal Behaviour 117:135-144.

Hsu, B.-Y., B. Doligez, L. Gustafsson, and S. Ruuskanen. $2019 a$. Transient growth-enhancing effects of elevated maternal thyroid hormones at no apparent oxidative cost during early postnatal period. Journal of Avian Biology 50:e01919.

Hsu, B.-Y., I. Verhagen, P. Gienapp, V. M. Darras, M. E. Visser, and S. Ruuskanen. 2019b. Data from: Between- and within-individual variation of maternal thyroid hormone deposition in wild great tits (Parus major). American Naturalist, Dryad Digital Repository, https://doi.org/10.5061/dryad.kp524f5.

Husby, A., L. E. B. Kruuk, and M. E. Visser. 2009. Decline in the frequency and benefits of multiple brooding in great tits as a consequence of a changing environment. Proceedings of the Roval Society B 276:1845-1854.

Kingma, S. A., J. Komdeur, O. Vedder, N. von Engelhardt, P. Korsten, and T. G. G. Groothuis. 2009. Manipulation of male attractiveness induces rapid changes in avian maternal yolk androgen deposition. Behavioral Ecology 20:172-179.

Lendvai, Á. Z., M. Giraudeau, V. Bókony, F. Angelier, and O. Chastel. 2015. Within-individual plasticity explains age-related decrease in stress response in a short-lived bird. Biology Letters 11:20150272.

Lendvai, Á. Z., J. Q. Ouyang, L. A. Schoenle, V. Fasanello, M. F. Haussmann, F. Bonier, and I. T. Moore. 2014. Experimental food restriction reveals individual differences in corticosterone reaction norms with no oxidative costs. PLoS ONE 9:e110564

Love, O. P., and T. D. Williams. 2008. The adaptive value of stressinduced phenotypes: effects of maternally derived corticosterone on sex-biased investment, cost of reproduction, and maternal fitness. American Naturalist 172:E135-E149.

Marshall, D. J., and T. Uller. 2007. When is a maternal effect adaptive? Oikos 116:1957-1963.

Martin, J. G. A., D. H. Nussey, A. J. Wilson, and D. Réale. 2011. Measuring individual differences in reaction norms in field and experimental studies: a power analysis of random regression models. Methods in Ecology and Evolution 2:362-374 
McNabb, F. M. A., and V. M. Darras. 2015. Thyroids. Pages 535-547 in C. G. Scanes, ed. Sturkie's avian physiology. 6th ed. Elsevier, London.

Mentesana, L., C. Isaksson, W. Goymann, M. N. Andersson, M. Trappschuh, and M. Hau. 2019. Female variation in allocation of steroid hormones, antioxidants and fatty acids: a multilevel analysis in a wild passerine bird. Journal of Avian Biology 50:e01859.

Müller, W., V. C. Goerlich, J. Vergauwen, T. G. G. Groothuis, and M. Eens. 2012. Sources of variation in yolk hormone deposition: consistency, inheritance and developmental effects. General and Comparative Endocrinology 175:337-343.

Nussey, D. H., E. Postma, P. Gienapp, and M. E. Visser. 2005. Selection on heritable phenotypic plasticity in a wild bird population. Science 310:304-306.

Nussey, D. H., A. J. Wilson, and J. E. Brommer. 2007. The evolutionary ecology of individual phenotypic plasticity in wild populations. Journal of Evolutionary Biology 20:831-844.

Okuliarova, M., T. G. G. Groothuis, P. Škrobánek, and M. Zeman. 2011. Experimental evidence for genetic heritability of maternal hormone transfer to offspring. American Naturalist 177:824-834.

Pant, K., and A. Chandola-Saklani. 1995. T3 fails to mimic certain effects of T4 in munia birds: physiological implications for seasonal timing. Comparative and Biochemical Physiology 111C:157-164.

Pérez, J. H., S. L. Meddle, J. C. Wingfield, and M. Ramenofsky. 2018. Effects of thyroid hormone manipulation on pre-nuptial molt, luteinizing hormone and testicular growth in male white-crowned sparrows (Zonotrichia leuchophrys gambelii). General and Comparative Endocrinology 255:12-18.

R Core Team. 2016. R: a language and environment for statistical computing. R Foundation for Statistical Computing, Vienna. https://www.R-project.org.

Reed, T. E., S. Wanless, M. P. Harris, M. Frederiksen, L. E. B. Kruuk, and E. J. A. Cunningham. 2006. Responding to environmental change: plastic responses vary little in a synchronous breeder. Proceedings of the Roval Society B 273:2713-2719.

Ruuskanen, S., V. M. Darras, B. de Vries, V. M. Visser, and T. G. G. Groothuis. 2016a. Experimental manipulation of food availability leads to short-term intra-clutch adjustment in egg mass but not in yolk androgen or thyroid hormones. Journal of Avian Biology 47:36-46.

Ruuskanen, S., V. M. Darras, M. E. Visser, and T. G. G. Groothuis. 2016b. Effects of experimentally manipulated yolk thyroid hormone levels on offspring development in a wild bird species. Hormones and Behavior 81:38-44.

Ruuskanen, S., P. Gienapp, T. G. G. Groothuis, S. V. Schaper, V. M. Darras, C. Pereira, B. de Vries, and M. E. Visser. 2016c. Heritable variation in maternally derived yolk androgens, thyroid hormones and immune factors. Heredity 117:184-190.

Ruuskanen, S., T. G. G. Groothuis, S. V. Schaper, V. M. Darras, B. de Vries, and M. E. Visser. 2016d. Temperature-induced variation in yolk androgen and thyroid hormone levels in avian eggs. General and Comparative Endocrinology 235:29-37.

Ruuskanen, S., and B.-Y. Hsu. 2018. Maternal thyroid hormones: an unexplored mechanism underlying maternal effects in an ecological framework. Physiological and Biochemical Zoology 91:904-916.

Schaper, S. V., A. Dawson, P. J. Sharp, P. Gienapp, S. P. Caro, and M. E. Visser. 2012. Increasing temperature, not mean temperature, is a cue for avian timing of reproduction. American Naturalist 179:E55-E69.

Schwabl, H. 1993. Yolk is a source of maternal testosterone for developing birds. Proceedings of the National Academv of Sciences of the USA 90:11446-11450.
Senapathi, D., M. A. C. Nicoll, C. Teplitsky, C. G. Jones, and K. Norris. 2011. Climate change and the risks associated with delayed breeding in a tropical wild bird population. Proceedings of the Royal Society B 378:3184-3190.

Shama, L. N. S. 2015. Bet hedging in a warming ocean: predictability of maternal environment shapes offspring size variation in marine sticklebacks. Global Change Biology 21:4387-4400.

Taff, C. C., and M. N. Vitousek. 2016. Endocrine flexibility: optimizing phenotypes in a dynamic world? Trends in Ecology and Evolution 31:476-488.

Tobler, M., M. Grandom, and M. I. Sandell. 2007. Maternal androgens in the pied flycatcher: timing of breeding and withinfemale consistency. Oecologia 151:731-740.

Too, H. C., M. Shibata, M. Yayota, V. M. Darras, and A. Iwasawa. 2017. Expression of thyroid hormone regulator genes in the yolk sac membrane of the developing chicken embryo. Lournal of Reproduction and Development 63:463-472.

Tschirren, B., J. Sendecka, T. G. G. Groothuis, L. Gustafsson, and B. Doligez. 2009. Heritable variation in maternal yolk hormone transfer in a wild bird population. American Naturalist 174:557-564.

van de Pol, M. 2012. Quantifying individual variation in reaction norms: how study design affects the accuracy, precision and power of random regression models. Methods in Ecology and Evolution 3:268-280.

van de Pol, M., and J. Wright. 2009. A simple method for distinguishing within-versus between-subject effects using mixed models. Animal Behaviour 77:753-758.

van Noordwijk, A., R. H. McCleery, and C. M. Perrins. 1995. Selection for the timing of great tit breeding in relation to caterpillar growth and temperature. Iournal of Animal Ecology 64:451-458.

Verhagen, I., P. Gienapp, V. N. Laine, E. M. van Grevenhof, A. C. Mateman, K. van Oers, and M. E. Visser. Forthcoming. Genetic and phenotypic responses to genomic selection for timing of breeding in a wild songbird. Functional Ecology, doi:10.1111/1365-2435.13360.

von Engelhardt, N., and T. G. G. Groothuis. 2011. Maternal hormones in avian eggs. Pages 91-127 in D. Norris and K. H. Lopez, eds. Hormones and reproduction of vertebrates. Vol. 4. Birds. Academic Press, London.

Westneat, D. F., M. I. Hatch, D. P. Wetzel, and A. L. Ensminger. 2011. Individual variation in parental care reaction norms: integration of personality and plasticity. American Naturalist 178:652-667.

Westneat, D. F., J. Wright, and N. J. Dingemanse. 2015. The biology hidden inside residual within-individual phenotypic variation. $\underline{\mathrm{Bi}}-$ ological Reviews 90:729-743.

Williams, T. D. 2008. Individual variation in endocrine systems: moving beyond the "tyranny of the golden mean." Philosophical Transactions of the Roval Society B 363:1687-1698.

2012. Physiological adaptations for breeding in birds. Princeton University Press, Princeton, NJ.

Williams, T. D., and T. G. G. Groothuis. 2015. Egg quality, embryonic development, and post-hatching phenotype: an integrated perspective. Pages 113-126 in D. C. Deeming and S. J. Reynolds, eds. Nests, eggs, and incubation: new ideas about avian reproduction. Oxford University Press, Oxford.

Wilson, A. J., D. Réale, M. N. Clements, M. M. Morrissey, E. Postma, C. A. Walling, L. E. B. Kruuk, and D. H. Nussey. 2010. An ecologist's guide to the animal model. Journal of Animal Ecology 79:13-26.

Associate Editor: Gregory E. Demas Editor: Russell Bonduriansky 